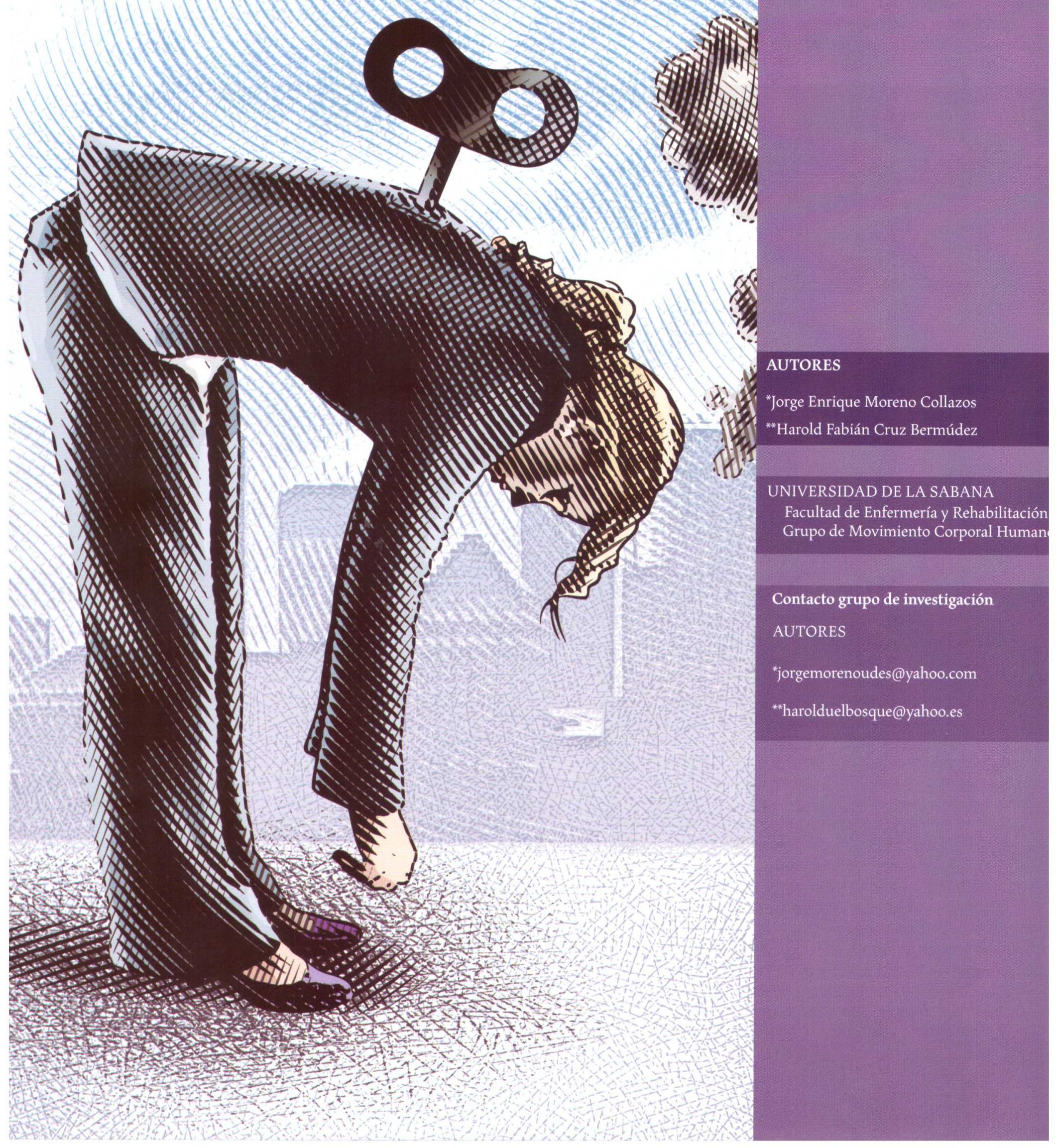




\section{CORRELACIÓN ENTRE LA FATIGA Y CALIDAD DE VIDA EN SUJETOS CON DIVERSOS TIPOS DE CÁNCER}

Fecha de recepción 6 de Julio de 2010 . Fecha de Aprobación 22 de Septiembre de 2010

\section{RESUMEN}

El objetivo del presente estudio fue realizar la correlación entre el índice de percepción de fatiga de Piper (IP) y la calidad de vida (CV) de sujetos con cáncer. La CV es la percepción del sujeto en relación con su entorno y el desarrollo de las actividades de la vida diaria (AVD) y el IP es una escala de percepción de fatiga ante la actividad física y las AVD. Por lo anterior se llevó a cabo un estudio no experimental descriptivo en un grupo poblacional de 40 sujetos con patología oncológica buscando la correlación entre la CV y el IP, para lo cual se aplicaron instrumentos de evaluación del índice de fatiga Escala de Piper y CV, Cuestionario EORTC. Para los datos obtenidos se aplicó la versión estadística del programa Statistical Package For The Social Sciences, V. 15, validados desde las técnicas previstas. La edad promedio fue $61 \pm 11.6$ años, el cáncer que más predominó fue de mama 32.5\%(n=13), cáncer de piel $20 \%(n=8)$ y próstata $10 \%(n=4)$. El IP promedio fue de $68.8 \pm 38,3$ (o-100 puntos), la variabilidad fue heterogénea ( $C V=24 \%$ ). Se encontró un puntaje alto de 2.88 (Media) en el dominio fatiga. La correlación entre la CV y el tiempo de evolución de la enfermedad fue de o,15 no se considera alta. Se observó una gran dispersión directamente proporcional: mientras la CV aumenta en puntaje (disminución de la $\mathrm{CV}$ ) el tiempo de la evolución de la enfermedad también aumenta. La correlación entre la CV y el IP presenta un valor medio $(0,49)$.

PALABRAS CLAVE

Fatiga, Calidad de Vida, Cáncer.

\section{ABSTRACT}

The aim of this paper was to determine the correlation between the Piper Fatigue Scale (IP) and quality of life (CV) of patients with cancer. The variable CV is the patient's perception in relation to his environment and the development of daily life activities and AVD. The variable IP is a scale of perception of fatigue to physical activity and ADL. This nonexperimental descriptive study was conducted in a group of 40 patients with oncologic pathology, seeking the correlation between the variables CV and IP, and applying instruments of evaluation of the fatigue scale, Piper Fatigue Scale and CV, EORTC Test. The obtained data was analyzed using the Statistical Package for the Social Sciences, V. 15, validated from provided techniques. The average age was $61 \pm 11.6$ years old. The types of cancer that occurred the most were breast cancer $32.5 \%(\mathrm{n}=13)$, skin cancer $20 \%(\mathrm{n}=8)$ and prostate cancer $10 \%(\mathrm{n}=4)$. The average for the variable IP was $68.8 \pm 38,3$ (Out of 100$)$, and the variability was heterogeneous $(\mathrm{CV}=24 \%$ ). It was found a high score of 2.88 (Media) in the domain of fatigue. The correlation between the variable $\mathrm{CV}$ and time of disease evolution was 0,15 , not considered high. A considerable directly proportional dispersion was observed, the more CV score increases ( $C V$ decreases), the more the time of the disease evolution increases. The correlation between the variables IP and CV was o,49, considered mean.

KEY WORDS

Fatigue, Quality of Life, Cancer 


\section{INTRODUCCIÓN}

$\mathrm{E}$ cáncer es una enfermedad conocida desde las antiguas civilizaciones. Su nombre deriva de la palabra kankros, que significa cangrejo y hace referencia a la similitud en que el animal y la enfermedad atrapan y destrozan a sus víctimas. Es una enfermedad con causas endógenas y exógenas que generan cambios estructurales y bioquímicos irreversibles e implican descendencia celular anómala (1).

En Colombia los tumores malignos representan da tercera causa de muerte y han aumentado su participación en la mortalidad general desde el $6 \%$, en la década de los sesenta, hasta el $15 \%$ en el año 2002 (2). El concepto de calidad de vida en el sujeto con patología oncológica, ha presentado considerables variaciones a través del tiempo (3). Primero, el término se refería al cuidado de la salud personal, después se adhirió la preocupación por la salud e higiene públicas y se extendió entonces a los derechos humanos, laborales y privados, lo cual continuó sumando la capacidad de acceso a los bienes económicos y finalmente se convirtió en la preocupación por la experiencia del sujeto sobre su vida social, su actividad cotidiana y su propia salud ( 4 ).

La European Organization for Research and Treatment of Cancer EORTC ha creado un grupo de estudio de calidad de vida, que ha llegado a un consenso sobre lo que debe incluir la evaluación de este concepto en el campo de la oncología: los síntomas físicos propios del cáncer, los debidos al tratamiento, los aspectos funcionales o capacidad para realizar actividades cotidianas como vestirse, comer, etc, los aspectos psicológicos y los aspectos sociales y familiares. Uno de los estudios más significativos se desarrolló en 12 países con diferentes culturas, incluida la española (5).

Según la OMS, la Calidad de Vida se define como la percepción que un individuo tiene de su lugar en la existencia, en el contexto de la cultura y del sistema de valores en los que vive y en relación con sus objetivos, sus expectativas, sus normas y sus inquietudes. Se trata de un concepto muy amplio que está influenciado de modo complejo por la salud física del sujeto, su estado psicológico, su nivel de independencia y sus relaciones sociales, así como su relación con los elementos esenciales de su entorno (6).

La fatiga es uno de los síntomas más referidos por los sujetos que sufren algún tipo de Cáncer, y sobre todo por aquellos que reciben algún tipo de terapia con médicamentos o invasiva para combatirlo, y se caracteriza principalmente por la aparición de síntomas tales como astenia, laxitud y debilidad, entre otros (7). Encontrar una definición integral de la fatiga es un desafío para los proveedores de cuidado a la salud, ya que al tener una etiología multifactorial resulta difícil elaborar su diagnóstico, evaluación y tratamiento (8).

La escala de percepción de fatiga fue desarrollada por Piper y colaboradores en 1998 en la Universidad de Nebraska. Se utiliza cuando las personas experimentan un sentido excesivo de cansancio inusual, cuando están enfermos, en tratamientos o se recuperan de enfermedad (9). Estudios relacionados con la percepción de fatiga indican la relación que esta muestra con la disminución de calidad de vida del individuo y su relación directa con su desempeñ en las actividades de la vida diaria (10).

Así mismo diversos autores como Servaes (11), han buscado cc nocer e investigar sobre las características de la fatiga en el pacient con cáncer, tratando de establecer una clara la definición de la mismé En ocasiones la han descrito como una preocupación fundamental a momento de realizar intervención en los sujetos con cáncer, los cuale hablan de fatiga crónica como desagradable, angustiosa, limitante d. la vida y de la actividad durante todo el día (11).

El fisioterapeuta, como un "profesional integral y generador d, adecuadas condíciones de vida", cumple un papel importante en e estudio, comprensión y manejo del Movimiento Corporal Humano como elemento esencial de la salud y bienestar del sujeto. Oriente sus acciones al mantenimiento, optímización o potencialización de. movimiento, así como a la prevención y recuperación de sus alteraciones y a la habilitación y rehabilitación integral de las personas, con el fin de optimizar su calidad de vida y contribuir al desarrollo social; y más aún cuando el movimiento corporal humano se ve afectado por patologías crónicas como en el caso del cáncer, que provoca un constante deterioro sistémico del organismo de las personas que lo padecen y por consiguiente un desmejoramiento de la calidad de vida, aun desconociéndose los factores intrínsecos y extrínsecos que se pueden relacionar en un proceso de rehabilitación integral $(12,13)$.

Diversos estudios han descrito la necesidad de relacionar variables tan importantes en el sujeto con cáncer, como son la calidad de vida y la percepción de fatiga en relación con la realización de las actividades de la vida diaria. Estudios realizados en Brasil nos muestran que a pesar de la mejora en la actividad física de los pacientes con cáncer por la intervención en aspectos terapéuticos, no se presenta una correlación significativa con la reducción de la fatiga, por lo cual hoy se presenta un estudio en el cual se realiza la correlación entre estas dos variables (14).

\section{METODOLOGÍA}

El estudio se realizó en el año 2009 en la fundación de Alivio contra el cáncer AVAC en Bucaramanga - Colombia. El diseño es no experimental, pues no existe manipulación de variables, y se parte de una observación de fenómenos de la Condición Física y su cuantificación respecto a percentiles establecidos en el ámbito de la salud terapéutica. Este estudio buscó especificar la calidad de vida y el nivel de fatiga en un grupo de pacientes con patología oncológica.

La investigación se realizó en el contexto del área de atención ambulatoria y hospitalización AVAC, institución pionera en Santander en brindar cuidados paliativos a enfermos agudos y crónicos de cáncer. La población fue de 108 enfermos atendidos en AVAC. Para el estudío se tomó una muestra no probabilística de cuarenta 
pacientes adultos de ambos sexos con patología oncológica, con edad de 25 años en adelante y sin compromiso de la esfera mental, lo que permitiría la autorización de la participación en el estudio. Para la conformación de la muestra se tomaron 20 pacientes internos en las sedes de hospitalización y zo pacientes externos en la modalidad de atención domiciliaria. Se tomaron como factores de exclusión pacientes con enfermedad cerebro vascular recientemente diagnosticados con cáncer cerebral, enfermedades cardiovasculares no controladas asociadas al cáncer, afecciones músculo esqueléticas a nivel de MMSS y/o MMII no controladas, hipoacusia, invidentes, posquirúrgicos recientes, y aquellos que se encontraban iniciando el tratamiento de quimioterapia y/o radioterapia para evitar confusiones en las variables tomadas en el estudio de la fatiga.

Para la definición de los dominios del instrumento de evaluación del índice de fatiga Escala de Piper y Calidad de Vida Cuestionario EORTC QLQ C-30 (European Organisation for Research and Treatment of Cancer quality of Life Questionnaire Core 30), con las áreas de funcionamiento que componen la calidad de vida, todas las escalas e ítems individuales se convierten en una puntuación de o a 100, donde a mayor puntaje hay más disminución de la calidad de vida. Se requirió inicialmente un consentimiento informado para la participación de los individuos en la investigación propuesta.

Así mismo se tuvieron en cuenta criterios de ingreso con un modelo inicial de Historia Clínica para tamizar la población en cuanto a antecedentes médicos, personales, familiares y tratamiento instaurado.

La evaluación en el paciente oncológico requirió valorar el nivel de fatiga, para lo cual fue incluido el índice de Piper el cual consta de 22 ítems repartidos en 4 subescalas: Conductual $(6$ ítems) pregunta 2-7, Afectiva ( 5 ítems) pregunta 8-12, Sensorial (5 items) pregunta 13-17, Cognitiva (6 ítems) pregunta 18-23. Se escoge la respuesta que mejor describa la fatiga que esté experimentando ese día. La calificación, según la Red Nacional Comprensiva del Cáncer, se delimita con una escala de o a 10 en que los pacientes reportan una fatiga leve (1-3), moderada $(4-6)$ o severa $(7-10)$.

En el área de la evaluación de la Calidad de Vida aplicada a enfermos de cáncer se tuvo en cuenta el cuestionario de calidad de vida de la Organización Europea para la Investigación en el tratamiento del Cáncer EORTC. Los resultados que se presentan a continuación surgen de la aplicación estadística del programa SPSS (Statistical Package For The Social Sciences, Versión 15), validados desde las técnicas previstas en el programa. Se digitó y depuró en Excel versión 2007 y el procesamiento se realizó en el programa Epi Info versión 3.5.1.

Para el análisis descriptivo de las variables cuantitativas se utilizó el promedio aritmético, el mínimo, el máximo, la desviación estándar y los cuartiles, y para determinar el grado de variabilidad se utilizó el coeficiente de variación. Para las variables cualitativas se utilizaron frecuencias absolutas y frecuencias relativas porcentuales. La correlación se determinó por medio del coeficiente de correlación de Pearson.

\section{RESULTADOS}

Características Generales: La edad promedio fue de $61 \pm 11.6$ años, la mínima edad fue de 40 y la máxima de 84 , la variabilidad fue medianamente homogénea ( $C V=19 \%$ ), el grupo de edad predominante fueron los mayores o iguales de 60 años (ver figura 1). El sexo predominante fue el femenino con un $72,5 \%(\mathrm{n}=29)$, lo cual tiene una presentación epidemiológica y estadística similar con otros estudios en donde el rango de edad se comporta de la misma manera (13). En relación con el sexo femenino, este se comporta según los estudios de la literatura científica en cáncer para Colombia (15).

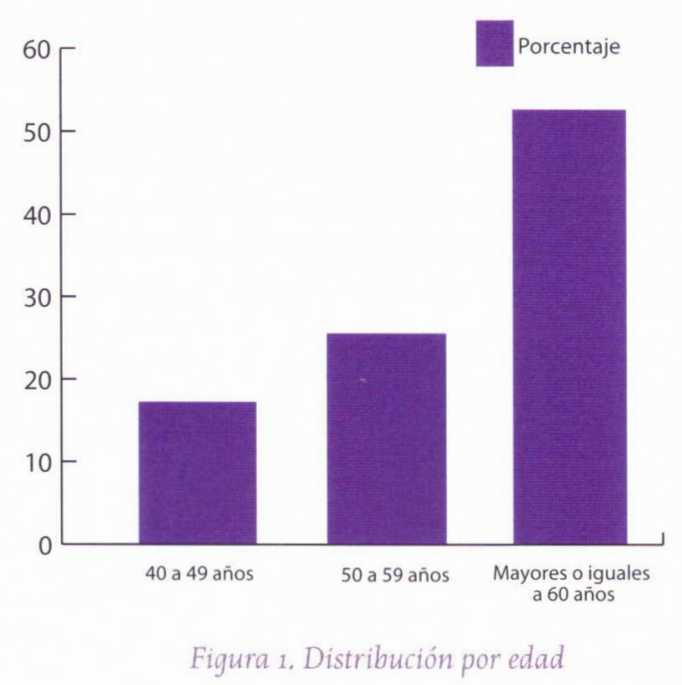

Dentro de las presentaciones clínicas de cáncer descritas en el estudio se encuentran: Cáncer de mama con un $32.5 \%(n=13)$ seguido del cáncer de piel con un $20 \%(n=8)$ y próstata con un $10 \%(n=4)$, el cáncer de cérvix, colon, pulmón, tiroides y útero se distribuyó en un $5 \%(\mathrm{n}=2)$ cada uno respectivamente, el cáncer de pene, leucemia, linfoma, mieloma múltiple y cáncer no especificado clínicamente se distribuyó en un $2.5 \%(n=1)$ cada uno respectivamente (ver tabla 1 ). Estos resultados concuerdan en descripción y presentación clínica con los tipos de cáncer más frecuentes en la población Colombiana (16).

\begin{tabular}{|c|c|}
\hline TIPOS DE CÁNCER & N. CASOS \\
\hline Cáncer Mama & 13 \\
\hline Cáncer Piel & 8 \\
\hline Cáncer Próstata & 4 \\
\hline Cáncer de Cérvix y Otros & 10 \\
\hline Cáncer de Pene y Otros & 5 \\
\hline Total Casos & 40 \\
\hline
\end{tabular}

El índice de fatiga promedio fue de $68.8 \pm 38,3$ con un mínimo de 23 , un máximo de 179 y una alta de $55.7 \%$, lo cual indica que hay una relación directa entre la situación clínica del paciente, su evolución en relación al cáncer y el índice de fatiga. Un mayor puntaje evidencia una 
mayor percepción de la fatiga (17). Así mismo hay que entender que según la literatura en algún momento de la evolución natural de la enfermedad el sujeto presenta fatiga (18).

El promedio de la calidad de vida en la última semana en los sujetos

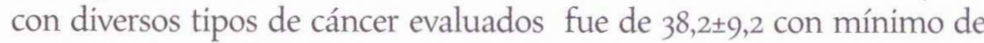
21 y un máximo de 61, en un escala de puntuación de $\mathrm{O}$ a 100, en la que a mayor puntaje peor calidad de vida global y con datos distribuidos de forma heterogénea.

\section{CORRELACIÓN}

La correlación entre la calidad de vida y el índice de fatiga fue de 0.487 , valor que puede considerarse medio. Se observó una dispersión baja y directamente proporcional: mientras aumenta el puntaje de la calidad de vida (esta disminuye) y el índice de fatiga aumenta, como puede observarse en la Figura 2. De esta manera se evidencia que fatiga es uno de los principales problemas que afecta la calidad de vida del sujeto con cáncer (19).

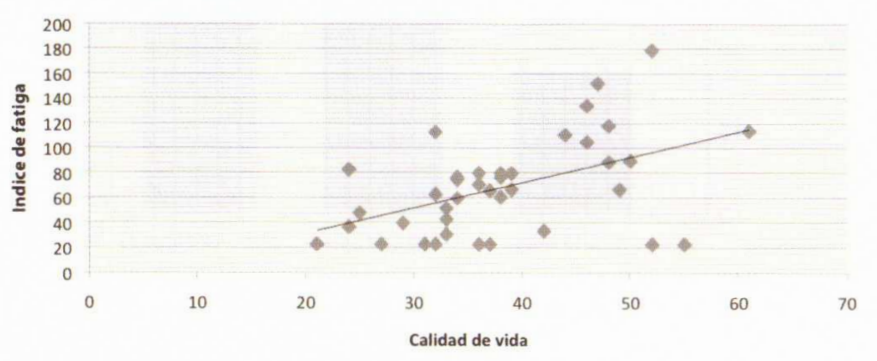

Figura 2. Dispersión entre calidad de vida e índice de fatiga

a.

La correlación entre la calidad de vida y el tiempo de evolución de la enfermedad fue de o,15, valor que no se considera alto. Se observó una gran dispersión, directamente proporcional: mientras la calidad de vida aumenta en puntaje (disminución de la calidad de vida) el tiempo de la evolución de la enfermedad también aumenta, como se aprecia en la Figura 3.

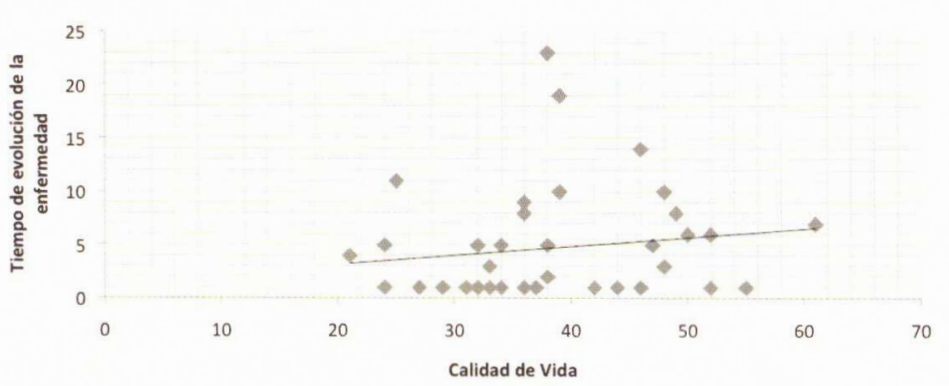

Figura 3. Dispersión entre calidad de vida y tiempo de evolución de la enfermedad
La aplicación de escalas tales como la EORTC en población cor cáncer, juega un papel importante en la comprensión general de lé calidad de vida de sujetos con diferentes tipos de cáncer, y sirve parć evaluar el impacto de la enfermedad en relación con sus actividades de la vida diaria y Calidad de Vida. Además, cada vez se hace más necesario que sea el mismo sujeto quien determine y mida su propic Calidad de Vida (20).

\section{CONCLUSIONES}

La calidad de vida sigue siendo unos de los aspectos más im. portantes para los profesionales proveedores de cuidados en salud: y por eso se hace necesario mejorar los patrones de actividad física y ejercicio, realizando intervenciones integrales en todos aquellos sujetos que presentan patología oncológica, si se desea optimizar e] cuidado de la salud y la calidad de vida (21).

La correlación entre la calidad de vida y el índice de fatiga se considera para el estudio alta. Se observó una baja dispersión directamente proporcional: mientras la calidad de vida aumenta en puntaje (disminuye la calidad de vida) y el índice de fatiga aumenta.

El promedio de la calidad de vida en la última semana en los sujetos con diversos tipos de cáncer evaluados fue homogéneo. Se evidenció que la fatiga es un factor determinante en la disminución de la calidad de vida Global del sujeto con cáncer al haber obtenido el puntaje más alto en los dominios evaluados de la escala $\operatorname{EORTC}(3,20)$.

Se evidencian de una manera clara las implicaciones en la actividad física entendidas como actividades de la vida diaria del sujeto con cáncer y su influencia en la disminución de la calidad de vida, por lo cual se entiende que la fatiga es uno de los principales problemas que afectan a estos sujetos.

Se debe valorar integralmente al sujeto con patología oncológica, buscando obtener datos más claros sobre las variabilidades entre la calidad de vida de la última semana y el índice de percepción de la fatiga.

En conclusión, se hace necesario cada vez más, en la intervención y la valoración fisioterapéutica, que sea el mismo sujeto quien determine, valore y mida su propia Calidad de Vida en relación con un proceso de rehabilitación integral. 


\title{
Referencia:
}

Las referencias a otras obras son una parte

muy importante en la literatura científica;

\author{
ya que estas permiten conocer más sobre los autores y \\ mantener vivas sus voces dentro del texto.
}

1. Aguilar J, Hernández M, Gómez M. Definición de cáncer. En: Cuidados paliativos e intervención psicosocial en enfermos terminales. Las Palmas de Gran Canaria: Ed. I.C.E.P.S.S; 1994; 164:13-4.

2. Departamento Administrativo Nacional de Estadística (DANE). Sistema de estadísticas vitales: Mortalidad en Colombia 2002 lbase de datos]. Bogotá: DANE; 2004.

3. Aaronson NK, Ahmedzai S. Bergman B, Bullinger M, Cull A, Duez NJ, et al. The european organization for research and treatment of cancer QLQ C-30: A quality of life instrument for use in international clinical trials in oncology. J Nat Cancer Inst. 1993; 85:365-76.

4. Holzner B, Kemmler G, Sperner-Unterweger B, Kopp M, Dünser M, Margreiter R et al. Quality of life measurement in oncology-a matter of the assessment instrument? Eur J Cancer 2001; 37(18): 2349-2356.

5. Aaronson NK. Assessing the quality of life of patients in cancer clinical trials. Eur. J. Cancer 1992; 28:1307-10.

6. Organización Mundial de la Salud. Cáncer. [en línea Febrero de 2006]. Consultado abril 292007 Available from: URL: http://www.who.int/ mediacentre/factsheets/fs297/es/index.html.

7. Miaskowski, C. Update on the assessment and management of cancerrelated fatigue. Principles and Practice of Supportive Oncology Updates 1998; 1(2):1-10.

8. Holler S. Fatigue in cancer patients: a descriptive study. Cancer Nursing $1991 ; 14(1): 13-19$.

9. Fatigue is redistributed by University of Bonn, Medical Center Información para profesionales de la salud. [en línea] [consultado 24 de marzo de 2007] Available from: URL://http://www.meb.uni-bonn. de/cancernet/spanish/304461.html.

10. Piper BE, Dibble SL, Dodd MJ, et al.: The revised Piper Fatigue Scale: psychometric evaluation in women with breast cancer. Oncol Nurs Forum $251998(4): 677-84$
11. Servaes P, Verhagen C, Bleijenberg G. Fatigue in cancer patients during and after treatment: prevalence, correlates and interventions. European Journal of Cancer 2002; 38: 27-43.

12. Hanna LR, Ávila PF, Meteer JD, Nicholas DR, Kaminsky LA. Theeffects of a Comprehensive Exercise Program on Physical Function, Fatigue, and Mood in Patients With Various Types of Cancer. ONCOLOGY NURSING FORUM 2008; 35 (3):461a-469.

13. Douglas E. Exercise in Cancer Patients. Physical Therapy Reviews 2005 ; 10: $71-78$.

14. Battaglini C, Bottaro M, Campbell J, Novaee J, Simão R. Actividad física y niveles de fatiga en pacientes portadores de cáncer. Rev Bras Med Esporte [online]. 2004, vol.10, n.2, pp. 98-104.

15. Gupta D, Lis C, Grutsch JF. The relationship between dyspnea and patient satisfaction with quality of life in advanced cancer. Supportive Care in Cancer 2007; 15(5): 533-538.

16. Pérez C, Meza E. Incidencia de cáncer en el Área Metropolitana de Bucaramanga, 2000-2004 Vol. 10 Número 3, Noviembre de 2007; 147-172

17. Sánchez R, Ballesteros M, Gómez A. Medición de la calidad de vida en ensayos clínicos de pacientes con cáncer. Un estudio bibliométrico Revista Colombiana de Cancerología 2009;13(1):29-34.

18. Suresh K. Reddy HA, Parsons AJ, Lynn P, Bruera E. Journal of Palliative Medicine. January 2009; $12(1): 29-36$.

19. Font A. Fatiga, expectativas y calidad de vida en cáncer. Psicooncologia $2004 ; 1(2-3): 45-46$

20. Arras J, Martines M, Manterota a, et al. La evaluación de la calidad de vida del paciente oncológico. El grupo de la calidad de vida de la EORTC. Psicooncologia, $2004 ; 1(1) ; 87-98$.

21. Hacker E. Trish Greene Memorial Quality-of-Life Lectureship Clinical Journal of Oncology Nursing. Vol 13, Num 1 - Exercise and Quality of Life 2008; 31-39.

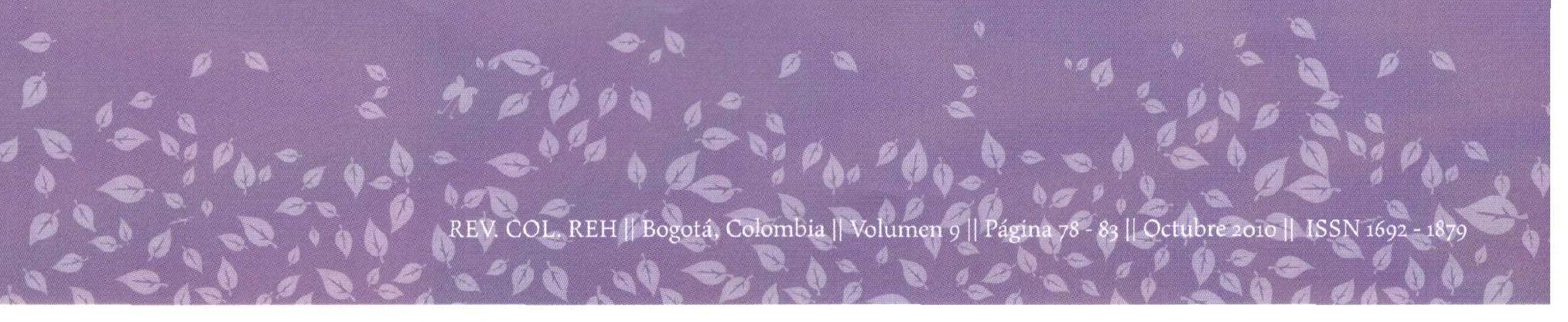

